\title{
A Scheme Design and Configuration Model of Mechanical Equipment Processing Technology based on Polychromatic Sets Theory
}

\author{
Gang Yang ${ }^{1, a}$ and Kangwei Chen ${ }^{2, b}$ \\ ${ }^{1}$ College of Applied Science and Technology, China University of Mining and \\ Technology, Xuzhou, Jiangsu Province, China PR, 221008 \\ ${ }^{2}$ Xuzhou Technician College,Xuzhou, Jiangsu Province, China PR, 221000 \\ ygcast@163.com, ${ }^{b}$ Ckw1980@163.com
}

\begin{abstract}
Various factors concerning design and producing need to be taken into account while designing and configuring the processing scheme of complex mechanical equipment. The quality of the processing scheme is directed related to the quality and function of the products. It is of great significance to make effective processing schemes for complex mechanical equipment processing. Thus, based on research and analysis of design and configuration of processing schemes of complex mechanical equipment, this paper presents a design and configuration model for designing processing scheme of complex mechanical equipment based on polychromatic sets theory. It obtains various designs and configuration schemes up to the standard of processing through using the concept of polychromatic graph and polychromatic set algorithm, and evaluates potential processing schemes based on multi-attribute fuzzy evaluation, to achieve the best processing scheme for complex mechanical equipment. Finally, this paper proves the efficacy and feasibility of the algorithm and the model through examining specific and concrete schemes.
\end{abstract}

Keywords: polychromatic sets theory;mechanical equipment; processing technology;scheme design; configuration

\section{Introduction}

With the rapid development of modern science and technology, especially that of computer technology and artificial intelligence, the design, development and processing of manufacturing industry has drastically changed. The development of Computer integrated manufacturing system, or CIMS, gradually becomes the mainstream for the technological development of manufacturing industry[1-2]. As the key stage in the development of complex equipment system, the design of processing schemes play an important role in the quality of complex equipment products. The research and development of Computer aided process planning, or CAPP, increase the importance of processing scheme in the manufacturing of products [3-4]. So far, scholars already researched on the processing schemes of complex mechanical products, and made achievements in research, as well as in engineering application, which proves to be efficient in practice [5-8]. However, research on the intelligent acquisition of processing schemes of complex mechanical products is limited. Sometimes processing schemes cannot be effectively obtained or the processing scheme is volatile, which all impair the manufacturing ability of the complex equipment system to a certain extent. Thus, based on studies of intelligent designing of processing schemes of complex equipment, this paper presents a design and configuration model of processing schemes of complex equipment based on Polychromatic sets theory, and offers a new solution for intelligent acquisition of processing schemes of complex equipment. 


\section{Polychromatic Graphs and Sets of Processing Schemes}

Unlike traditional sets, polychromatic sets can not only indicate the names of their elements, but also color these elements and thus express their property. Thus, with the ability to extend and adapt, they can be used to study different subjects based on the same mathematical model. This paper intends to study the design of processing schemes of complex equipment using Polychromatic sets theory. All procedures in the process will be colored as elements in the polychromatic graph of processing scheme design. In the meantime, the design and producing of complex equipment, along with processing and technical requirement, will each be designated with a fixed color, thus forming a polychromatic graph $\mathrm{G}$ where processing scheme contour matrix $[A \times F(A)]$ interacts with operator.

All procedures $a_{i}$ that are generated by a processing scheme are the basic elements of polychromatic set $A$. Thus, the basic arithmetic formula of the polychromatic set of a processing scheme would be:

$$
A=\left(a_{1}, a_{2}, \cdots, a_{i}, \cdots, a_{n}\right)
$$

The generation of a processing scheme requires certain designing and technical conditions, and has certain limitations. These conditions and limitations make up fixed colors of processing schemes $F(A)$, with the arithmetic formula:

$$
F(A)=\left(F_{1}, F_{2}, \cdots, F_{j}, \cdots, F_{m}\right)
$$

If element $a_{i}$ in the polychromatic set can match the corresponding element in $F(A)$, then $F\left(a_{i}\right)$ will be called "individual color", its value being Boolean vector. Thus, the processing scheme contour matrix $[A \times F(A)]$ based on the relation of the individual color $F\left(a_{i}\right)$ and the fixed color $F(A)$ will have the following formula:

$$
\left\|a_{i j}\right\|_{A \times F(A)}=\left|\begin{array}{ccccc}
a_{11} & \cdots & a_{1 j} & \cdots & a_{1 m} \\
\vdots & \vdots & \vdots & \vdots & \vdots \\
a_{i 1} & \cdots & a_{i j} & \cdots & a_{i m} \\
\vdots & \vdots & \vdots & \vdots & \vdots \\
a_{n 1} & \cdots & a_{n j} & \cdots & a_{n m}
\end{array}\right|_{n \times m}
$$

In this formula, if $F_{j} \in F\left(a_{i}\right)$, meaning $F\left(a_{i}\right)$ meets the requirement of $F_{j}$ from $F(A)$, then $a_{i j}=1$, otherwise, $a_{i j}=0$.

Polychromatic graph $\mathrm{G}$ is the graphical representation of polychromatic set, which shows the executing order of the elements in the polychromatic set. Its arithmetic model is:

$$
G=\left(F(G), S_{A}, S_{C}\right)
$$

In this formula, $F(G)$ is the fixed color of the polychromatic set.

$S_{A}$ stands for the polychromatic set of nodes in the polychromatic graph G.. Its arithmetic model is: 


$$
S_{A}=(A, F(A), F(a),[A \times F(A)],[A \times F(a)],[A \times A(F)])(5)
$$

$S_{C}$ represents the polychromatic set of sides in the polychromatic graph G. Its arithmetic model is:

$$
S_{C}=(C, F(C), F(c),[C \times F(C)],[C \times F(c)],[C \times C(F)])
$$

Particularly, if neither the nodes nor the sides of the polychromatic graph $\mathrm{G}$ is colored, then it would be an ordinary graph of a processing scheme. Thus, an ordinary graph is an exception of a polychromatic graph $\mathrm{G}$.

\section{Processing Technology Design based on Polychromatic Sets Theory}

\subsection{Generation of Processing Technology Path based on Polychromatic Sets Theory}

After constructing the contour matrix $[A \times F(A)]$, a processing scheme based on the contour $[A \times F(A)]$ should be generated, which means a processing procedure would be acquired. The algorithm of generating a processing procedure is:

First, ascertain the contour $F(P)$ of a product based on the processing and technical requirements, and the limitation.

Second, start analyzing from the first step, study any line $t$ of the readily available contour $[A \times F(A)]$ based on formula (7), and acquire contour $F(P)_{i}^{t}$ that meets a certain processing requirement.

$$
\left\{\begin{array}{l}
F(P)_{i}^{t}=F(A) \wedge F\left(a^{t}\right) \\
\exists F(P)_{i}^{t}=1, \text { then } F\left(a_{i}^{t}\right)=F\left(a^{t}\right)
\end{array}\right.
$$

In this formula, $i$ represents the corresponding operator.

Third, if the operator fits into formula (8), it means operator $k$ that has the same function as operator $i$ exits in the available operators. If a total number of $s$ operators have the same function, it means a total number of $s-1$ processing procedures can be generated.

$$
\exists F(P)_{i}=F(P)_{i-1}, \text { then } F\left(a_{i}^{t}\right)=F\left(a_{k}^{t}\right)
$$

Fourth, a processing scheme contour $F(P)_{i}$ can be generated with a total number of $i-1$ available operators and formula (9):

$$
F(P)_{i}=F(P)_{i-1} \vee F\left(a_{i}^{t}\right)
$$

Fifth, repeat the above steps, acquire all the lines of contour matrix $[A \times F(A)]$, and examine whether the contour is encapsulated and feasible. If the value of any element in $F(P)_{A}$ is 1 , then it is. 


$$
\left\{\begin{array}{l}
F(P)_{A}=F(P)_{i} \wedge \bar{F}(P) \\
\exists \forall\left(\left(p \in F(P)_{A}\right) \wedge p=1\right), \text { then } F(P)_{A}=\text { true }
\end{array}\right.
$$

Sixth, all operators up to the standard can be acquired. Search the polychromatic graph $\mathrm{G}$ with all the acquired operators, and figure out the sub-graph that contains the above operators. In the meantime, consider operators in the third step that have the same function, and form multiple processing schemes up to the requirements.

\subsection{Optimization of Processing Technology based on Fuzzy Analysis}

Since the multiple processing schemes acquired from 3.1 are not the ultimate ones, during the process of optimizing, the corresponding optimizing quality parameter is usually not a certain value. Thus, this paper adopts fuzzy analysis [14-16] to optimize the multiple processing schemes with the following steps:

First, assuming that a total number of $M$ processing schemes are acquired from 3.1, a scheme set $\mathrm{C}$ can be formed:

$$
C=\left(C_{1}, \cdots, C_{2}, \cdots, C_{M}\right)
$$

Second, consult designing experts and engineer designers in the field to acquire a set of optimizing parameter $U$ :

$$
U=\left(U_{1}, \cdots, U_{2}, \cdots, U_{N}\right)
$$

Third, allocate weight to different parameter in the set $U$, and obtain a corresponding sequence of weight $W$ :

$$
W=\left(W_{1}, \cdots, W_{2}, \cdots, W_{N}\right)
$$

Fourth, assuming the value of the processing scheme $C_{i}$ relative to optimizing parameter $U_{j}$ is $v_{i j}=\left[v_{i j}^{a}, v_{i j}^{b}\right], v_{i j}^{a} \leq v_{i j}^{b}$, when it is a parameter for efficiency, its standard value is $\bar{v}_{i j}=\left[\begin{array}{cc}-a & -b \\ v_{i j} & , v_{i j}\end{array}\right]$ :

$$
\bar{v}_{i j}=\left[\begin{array}{cc}
-a & -b \\
v_{i j} & v_{i j}
\end{array}\right]=\left(v_{i j}^{a} / \max _{1 \leq j \leq N}\left(v_{i j}^{b}\right), v_{i j}^{b} / \max _{1 \leq j \leq N}\left(v_{i j}^{b}\right)\right)
$$

Fifth, assuming the value of the processing scheme $C_{i}$ relative to optimizing parameter $U_{j}$ is $v_{i j}=\left[v_{i j}^{a}, v_{i j}^{b}\right], v_{i j}^{a} \leq v_{i j}^{b}$, when it is a parameter for cost, its standard value is $\bar{v}_{i j}=\left[\begin{array}{cc}-a & -b \\ v_{i j}, & v_{i j}\end{array}\right]$ :

Thus, a contour B that shows the relation of the set of processing scheme $C$ and the set of optimizing parameters $U$ can be formed:

$$
\boldsymbol{B}=\left|\begin{array}{ccccc}
- & & - & \cdots & - \\
v_{11} & \cdots & \bar{v}_{1 j} & \cdots & v_{1 N} \\
\vdots & \cdots & \vdots & \cdots & \vdots \\
- & \cdots & - & & - \\
v_{i 1} & \cdots & \bar{v}_{i j} & \cdots & \bar{v}_{i N} \\
\vdots & \cdots & \vdots & \cdots & \vdots \\
- & \cdots & - & \cdots & - \\
v_{M 1} & \cdots & \bar{v}_{M j} & \cdots & \bar{v}_{M N}
\end{array}\right|_{M \times N}
$$


Sixth, construct an ideal parameter value of the set of processing schemes $C$ relative to the set pf optimizing parameters $U v_{i j}^{0}=\left[v_{i j}^{0 a}, v_{i j}^{0 b}\right]$ :

$$
v_{i j}^{0}=\left[v_{i j}^{0 a}, v_{i j}^{0 b}\right]=\left\lceil\max _{1 \leq j \leq M}\left(\begin{array}{c}
-a \\
v_{i j}
\end{array}\right), \max _{1 \leq j \leq M} v_{i j}^{-b}\right\rceil
$$

Seventh, the fuzzy distance $U_{j}^{0}$ can be constructed as follows:

$$
D_{i j}=\left(\left|v_{i j}^{0 a}-v_{i j}^{a}\right|^{2}+\left|v_{i j}^{0 b}-v_{i j}^{b}\right|^{2}\right)^{\frac{1}{2}} / \sqrt{2}
$$

Eighth, the weight given to different parameters considered, the weighted fuzzy distance $D_{i}^{W}$ needs to be formed, as well as a sequence $D^{W}$ :

$$
\begin{aligned}
D^{W}= & B \otimes W^{T}=\left[D_{1}^{W}, \cdots, D_{i}^{W}, \cdots, D_{M}^{W}\right] \\
& =\left|\begin{array}{ccccc}
- & - & \cdots & - \\
v_{11} & \cdots & v_{1 j} & \cdots & v_{1 N} \\
\vdots & \cdots & \vdots & \cdots & \vdots \\
- & \cdots & - & \cdots & - \\
v_{i 1} & \cdots & v_{i j} & \cdots & \bar{v}_{i N} \\
\vdots & \cdots & \vdots & \cdots & \vdots \\
- & \cdots & - & \cdots & - \\
v_{M 1} & \cdots & v_{M j} & \cdots & \bar{v}_{M N}
\end{array}\right|_{M \times N}\left|\begin{array}{c}
W_{1} \\
\vdots \\
\vdots \\
\vdots \\
W_{N}
\end{array}\right|_{N \times 1}
\end{aligned}
$$

Ninth, according to the optimizing principle, if processing scheme $C_{i}$ is the best, then its corresponding fuzzy distance $D_{i}^{W}$ should be the shortest. Thus, the best scheme $C_{k}$ can be obtained through forming the formula (20):

$$
D_{0}^{W}=\min \left(D_{1}^{W}, \cdots, D_{i}^{W}, \cdots, D_{M}^{W}\right)=D_{k}^{W}, 1 \leq k \leq M
$$

\section{Case Analysis and Verification}

This part analyzes and illustrates the theory, model and method above with the example of making the processing scheme of a thin-walled cylinder workpiece. After analysis of

\begin{tabular}{|c|c|c|c|}
\hline symbol & meaning & symbol & meaning \\
\hline contour $F_{1}$ & $\begin{array}{l}\text { Processing the outside } \\
\text { cylinder surface }\end{array}$ & contour $F_{15}$ & $\begin{array}{l}\text { The length of shaft blank } \\
0-500\end{array}$ \\
\hline contour $F_{2}$ & Processing the traverse plane & contour $F_{16}$ & $\begin{array}{c}\text { The length of shaft blank } \\
500-1000\end{array}$ \\
\hline contour $F_{3}$ & $\begin{array}{l}\text { Processing the inside } \\
\text { cylinder surface }\end{array}$ & contour $F_{17}$ & $\begin{array}{l}\text { The length of shaft blank } \\
1000-1500\end{array}$ \\
\hline contour $F_{4}$ & Processing the groove & operator $a_{1}$ & Rough turning \\
\hline
\end{tabular}
processing requirements and procedures, corresponding contours and operators can be obtained. Table 1 shows the meaning of relevant symbols of contours and operators.

Table 1. Symbols and Meanings of Process Operators and Contours in the Design Scheme 


\begin{tabular}{|l|c|l|c|}
\hline contour $F_{5}$ & Accuracy level 4-7 & operator $a_{2}$ & Semi finish turning \\
\hline contour $F_{6}$ & Accuracy level 8-10 & operator $a_{3}$ & Finish turning \\
\hline contour $F_{7}$ & Accuracy level 11-13 & operator $a_{4}$ & Grinding \\
\hline contour $F_{8}$ & Roughness 0.01-1.25 & operator $a_{5}$ & Rough milling \\
\hline contour $F_{9}$ & Roughness 1.25-5 & operator $a_{6}$ & Finish milling \\
\hline contour $F_{10}$ & Roughness 5-10 & operator $a_{7}$ & Drilling \\
\hline contour $F_{11}$ & Roughness over 10 & operator $a_{8}$ & Reaming \\
\hline contour $F_{12}$ & $\begin{array}{c}\text { Outer diameter of shaft blank } \\
0-40\end{array}$ & operator $a_{9}$ & Rough boring \\
\hline contour $F_{13}$ & $\begin{array}{c}\text { Outer diameter of shaft blank } \\
40-50\end{array}$ & operator $a_{10}$ & Finish boring \\
\hline contour $F_{14}$ & $\begin{array}{c}\text { Outer diameter of shaft blank } \\
50-1000\end{array}$ & & \\
\hline
\end{tabular}

Figure 1 shows the relation among operators, namely polychromatic graph of operators. In this graph, $a_{0}$ indicates the unified blanking procedure.

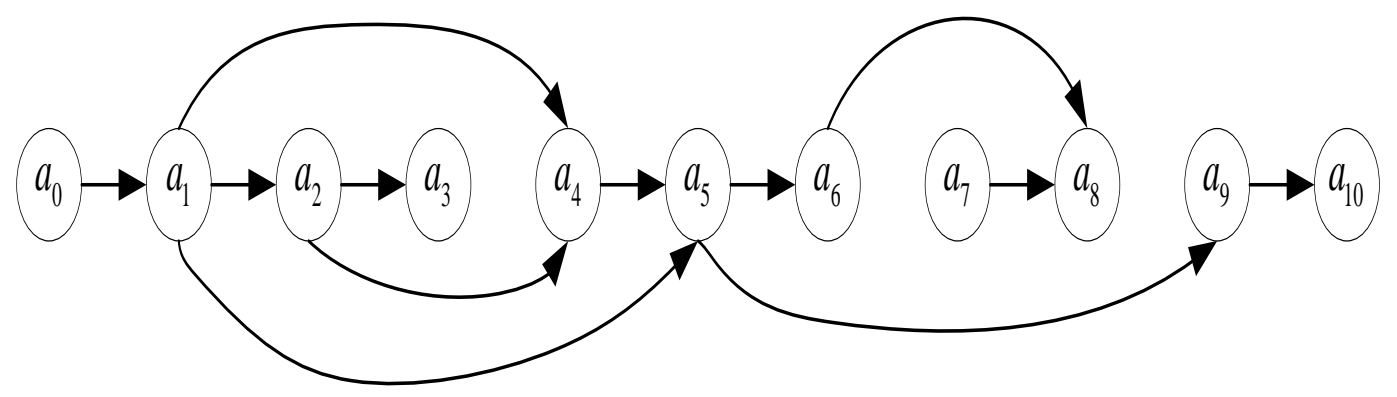

Figure 1. Relationship Between Process Operators

Thus, the contour matrix can be formed, as in Table 2.

Table 2. Contour Matrixes of the Design Scheme

\begin{tabular}{|c|c|c|c|c|c|c|c|c|c|c|c|c|c|c|c|c|c|}
\hline & $F_{1}$ & $F_{2}$ & $F_{3}$ & $F_{4}$ & $F_{5}$ & $F_{6}$ & $F_{7}$ & $F_{8}$ & $F_{9}$ & $F_{10}$ & $F_{11}$ & $F_{12}$ & $F_{13}$ & $F_{14}$ & $F_{15}$ & $F_{16}$ & $F_{17}$ \\
\hline$a_{1}$ & 0 & 0 & & & & & & & & & 0 & 0 & 0 & 0 & 0 & 0 & 0 \\
\hline$a_{2}$ & 0 & 0 & & & & 0 & 0 & & 0 & 0 & & 0 & 0 & 0 & 0 & 0 & 0 \\
\hline$a_{3}$ & & & & & 0 & 0 & & 0 & & & & 0 & 0 & 0 & 0 & 0 & 0 \\
\hline$a_{4}$ & & & & & 0 & 0 & & 0 & & & & 0 & 0 & 0 & 0 & 0 & 0 \\
\hline$a_{5}$ & & & & 0 & & & 0 & & 0 & & & 0 & 0 & 0 & 0 & 0 & 0 \\
\hline$a_{6}$ & & & & 0 & 0 & 0 & & 0 & & & & 0 & 0 & 0 & 0 & 0 & 0 \\
\hline$a_{7}$ & & & 0 & & & & 0 & & & & 0 & 0 & 0 & 0 & 0 & 0 & 0 \\
\hline$a_{8}$ & & & 0 & & & 0 & & & & 0 & 0 & 0 & 0 & 0 & 0 & 0 & 0 \\
\hline$a_{9}$ & & & 0 & & 0 & 0 & & & & 0 & 0 & 0 & 0 & 0 & 0 & 0 & 0 \\
\hline$a_{10}$ & & & 0 & & 0 & 0 & & 0 & 0 & & & 0 & 0 & 0 & 0 & 0 & 0 \\
\hline
\end{tabular}


The processing of thin-walled cylinder workpiece includes that of outside cylinder surface, inside cylinder surface and two traverse planes. The next part illustrates the model and algorithm through the example of processing outside cylinder surface. The processing requires accuracy level to be 6 , roughness $6.3 \mu \mathrm{m}$, maximum outer diameter of shaft blank $240 \mathrm{~mm}$, the maximum length of shaft blank $900 \mathrm{~mm}$. Then the corresponding product contour based on the polychromatic graph in Figure 1 will be:

$$
F(P)=(1,0,0,0,1,0,0,0,0,1,0,0,0,1,0,0,1)
$$

According to the algorithm of generating processing procedures, and the polychromatic graph in Figure 1, two schemes can be formed: $C_{1}=\left(a_{1}, a_{2}, a_{3}\right)$ and $C_{2}=\left(a_{1}, a_{2}, a_{4}\right)$. In order optimize these two schemes, values need be generated in Table 3 after consulting experts in the field on optimizing parameters and corresponding values.

Table 3. Optimizing Parameters and Values

\begin{tabular}{|c|c|c|c|}
\hline \multirow{2}{*}{} & \multirow{2}{*}{ weight } & \multicolumn{2}{|c|}{ values } \\
\cline { 3 - 4 } & & scheme $C_{1}$ & scheme $C_{2}$ \\
\hline time & 0.40 & 2.60 & 1.52 \\
\hline efficiency & 0.25 & $0.90-0.95$ & $0.75-0.85$ \\
\hline cost & 0.15 & 101 & 120 \\
\hline feasibility & 0.20 & $0.80-0.85$ & $0.85-0.90$ \\
\hline
\end{tabular}

The standard matrix of values can be acquired according to 3.2:

$$
\boldsymbol{B}=\left|\begin{array}{llll}
0.585 & 0.947-1.000 & 1.000 & 0.889-0.945 \\
1.000 & 0.789-0.895 & 0.842 & 0.945-1.000
\end{array}\right|_{2 \times 4}
$$

The fuzzy distance:

$$
\boldsymbol{D}=\left|\begin{array}{cccc}
0.415 & 0 & 0 & 0.056 \\
0 & 134 & 0.158 & 0
\end{array}\right|_{2 \times 4}
$$

The weight of different parameters considered, the sequence of fuzzy distance can be acquired: $D^{W}=\left(D_{1}^{W}, D_{2}^{W}\right)=(0.177,0.057)$. Apparently, $D_{1}^{W}<D_{2}^{W}$, meaning under the optimizing principle, scheme $C_{2}=\left(a_{1}, a_{2}, a_{4}\right)$ has better efficiency and produces better quality products. Thus it can be chosen as the processing scheme. The processing schemes of other surfaces are similar.

\section{Conclusion}

This paper analyzes the design and configuration of processing schemes of complex mechanical equipment, and presents a design and configuration model for designing processing of complex mechanical equipment based on Polychromatic sets theory. Through constructing contour matrix and the polychromatic graph of operators and acquiring clear processing procedures, it can acquire various potential processing schemes. In the meantime, this paper also studies the optimization of various schemes based on fuzzy mathematics theory, and offers corresponding fuzzy optimizing algorithm, to ensure the astringency of schemes acquired based on Polychromatic sets theory. The design and configuration of processing schemes features simple algorithm, and can be conducted by computers. Thus it offers a new solution for CAPP of complex mechanical equipment processing schemes. Its efficacy and feasibility are both verified through real examples. 


\section{References}

[1] S. Qiu, S. Fan and C. Gao, "Research on the Method of Comprehensive Evaluation for Workshop Facilities Layout Planning in CIMS", Modern Manufacturing Engineering, vol. 4, (2014).

[2] S. Qiu, Y. Lu and Y. Lu, "The Design and Implementation of Electronic Product Quality Reliability Management System in CIMS", Manufacturing Automation, vol. 3, no. 35, (2013).

[3] J. Yang, "The Application of CAPP in the Modern Enterprise", China Metalforming Equipment \&Manufacturing Technology, vol. 4, (2013).

[4] Q. Zhang and S. Liu, "3D CAPP Integrated System Development Based on B/S", Aeronautical Manufacturing Technology, vol. 3, (2013).

[5] L. Jiang, S. Zhao and Z. Li, "Punching Process Planning Modeling Based on Polychromatic Sets Theory, Computer Integrated Manufacturing Systems", vol. 7, no. 12, (2006).

[6] M. Shi, "The CAPP of Motor End Cover Components", Manufacturing Automation, vol. 7, no. 31, (2009).

[7] Y. Wang, "Artificial Intelligence Application in CAPP”, Automation \& Instrumentation, vol. 4, (2012).

[8] J. Chen, "Research on CAPP in Application of Mould Numerical Control Machining", Coal Mine Machinery, vol. 10, no. 34,(2013).

[9] X. Gao, Y. Li and M. Yang, "Management and Allocation of Workflow Resource Based on Isolated Operation of PS", Computer Integrated Manufacturing Systems, vol. 9, no. 17, (2011).

[10] S. Tong and X. Xu, "Relationships Network Model of Factors Influencing Manufacturing Quality Based on Polychromatic sets", Computer Integrated Manufacturing Systems, vol. 7, no. 15, (2009).

[11] X. Gao and Z. Li, "Workflow Modeling Method Based on UML \& Polychromatic Sets Theory", Computer Integrated Manufacturing Systems, vol. 7, no. 12, (2006).

[12] X. Gao, Z. Li and S. Zhao, "Study on Modeling and Reasoning Technology of Conceptual Design Based on Polychromatics", China Mechanical Engineering, vol. 3, no. 17, (2006)

[13] S. Wang, Y. Li and F. Chen, "Process Planning and Resource Allocation in Aircraft Connection Assembly Based on Polychromatic Sets", Computer Integrated Manufacturing Systems, vol. 2, no. 19, (2013).

[14] T. Wang, A. Yang and S. Zhong, "Multi-attribute Extension Fuzzy Optimized Decision-making Model of Scheme Design", Technical Gazette, vol. 2, no. 21, (2014).

[15] C. Rao, X. Xiao and P. Jin, "Novel Combinatorial Algorithm for the Problems of Fuzzy Grey Multi-attribute Group Decision Making”, Journal of Systems Engineering and Electronics, vol. 4, no. 18, (2007)

[16] P. Wang, D. Zhang and B. Chen, "Evaluation of Multi-process Plans Based on Fuzzy Comprehensive Evaluation and Grey Relational Analysis", Journal of Aerospace Power, vol. 9, no. 27 (2012).

\section{Authors}

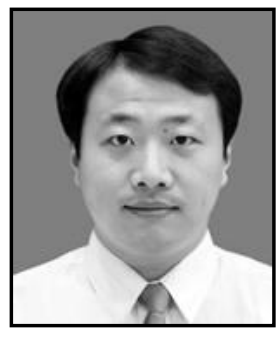

Gang Yang, aged 35, Doctor's degree, he is working in College of Applied Science and Technology, China University of Mining and Technology. His major field is advanced design and manufacturing method. The address is College of Applied Science and Technology, CUMT, Xuzhou, Jiangsu Province, China PR.

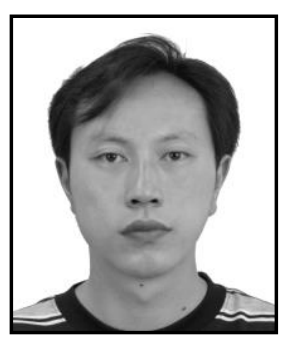

Kangwei Chen, Aged 34, Master's degree, He is working in Xuzhou Technician College. His major field is design and manufacturing of mechanical equipment. The address is Xuzhou Technician College, Xuzhou, Jiangsu Province, China PR. 